\title{
Hipertensão arterial e osteoporose no processo do envelhecimento: uma revisão de literatura
}

\author{
Luana Decian Saquet"; Grazielle Castagna Cezimbra Weis"; Bruna de Moraes Rossato*; \\ Cassiano Boessio Vizzoto"; Cristiana Basso"; Adriane Cervi Blümke***
}

\section{Resumo}

As doenças crônicas não transmissíveis são umas das principais causas de morte no Brasil. O objetivo deste estudo foi realizar uma revisão de literatura avaliando a prevalência, os fatores de risco, a prevenção e o tratamento de duas doenças envolvidas no processo de envelhecimento, a hipertensão e a osteoporose. Foi realizado busca nas bases de dados Biblioteca Virtual em Saúde (BVS) e Periódicos da Coordenação de Aperfeiçoamento de Pessoal de Nível Superior (Capes), cujos termos de busca foram "doença crônica", "idoso", "hipertensão" ou "osteoporose". Foram incluídos artigos originais e artigos de revisão em português ou em inglês, e excluiu-se da pesquisa artigos que não estivessem disponíveis online gratuitamente na íntegra. Foram encontrados 1393 artigos, sendo selecionadas para o estudo 26 publicações nacionais e internacionais, divulgadas entre os anos de 2004 e 2014. Como resultados, observou-se que a hipertensão e a osteoporose consistem em doenças de elevada prevalência entre os idosos, principalmente entre as mulheres. Fatores genéticos e ambientais, como o tabagismo, o etilismo, o sedentarismo e a alimentação constituem nos principais fatores de risco dessas doenças. A prevenção dessas doenças é baseada em cuidados com os fatores de risco, como a prática de exercícios físicos e uma alimentação rica em frutas e verduras, e pobre em sal, no caso da hipertensão. O tratamento da osteoporose e da hipertensão usualmente ocorre por intervenção cirúrgica e farmacológica, respectivamente. Os resultados encontrados neste estudo contribuem para o melhor entendimento sobre essas doenças, auxiliando na busca por um envelhecimento saudável.

Palavras-chave: Envelhecimento. Hipertensão. Osteoporose.

\section{Introdução}

A população brasileira está em processo de envelhecimento. Conforme dados do XII Recenseamento Geral do Brasil do Censo 2010, realizado pelo Instituto Brasileiro de Geografia e Estatística (IBGE), a proporção de idosos

* Graduação em Nutrição pelo Centro Universitário Franciscano, Santa Maria (RS). E-mail: luana.258@ hotmail.com; grazielle.castagna@gmail.com; brunamrossato@gmail.com;cassibv@hotmail.com

** Nutricionista. Mestre em Ciência e Tecnologia dos Alimentos pelo Programa de Pós-Graduação em Ciência e Tecnologia dos Alimentos da Universidade Federal de Santa Maria (UFSM). Docente do Departamento de Ciências da Saúde do Centro Universitário Franciscano, Santa Maria (RS). E-mail: cristiana@unifra.br

*** Nutricionista. Mestre em Ciência da Nutrição pela Universidade Federal de Viçosa. Docente do Departamento de Ciências da Saúde do Centro Universitário Franciscano, Santa Maria (RS). E-mail:adriblumke@yahoo. com.br

$\rightarrow$ http://dx.doi.org/10.5335/rbceh.v15i1.6554 
aumentou de 5,9\% em 2000 para 7,4\% em 2010 (IBGE, 2010). Estima-se que em 2025, o Brasil terá a sexta maior população de idosos no mundo (CAMARANO, 2006).

De acordo com a Organização Mundial da Saúde (OMS), as doenças crônicas não transmissíveis (DCNTs) são as doenças mais comuns na população idosa (ORGANIZAÇÃO MUNDIAL DA SAÚDE, 2003).

Mundialmente, as DCNTs são responsáveis por $60 \%$ das mortes e incapacidade. No Brasil, este número aumenta, e no ano de 2014, as doenças crônicas corresponderam a $74 \%$ de todas as mortes ocorridas no país (ORGANIZAÇÃO MUNDIAL DA SAÚDE, 2014).

A hipertensão arterial e a osteoporose são doenças consideradas predominantes entre as DCNTs, apresentando elevada prevalência na população idosa (SOCIEDADE BRASILEIRA DE CARDIOLOGIA, 2010).

A hipertensão arterial possui como característica níveis elevados e sustentados de pressão arterial, sendo considerada um problema de saúde pública pela complexidade dos recursos necessários para seu tratamento (VIEIRA et al., 2016; MACHADO et al., 2017).

Já a osteoporose é uma doença que ocorre devido à diminuição progressiva da massa óssea, consequentemente tornando os ossos mais frágeis e propensos à fraturas (ANDRADE, 2015). Devido ao envelhecimento da população mundial, a osteoporose obteve uma incidência crescente nas últimas décadas. Por isso, a detecção precoce da osteoporose ajuda a alertar os profissionais de saúde para o tratamento (REBELO, 2016).

Assim, devido ao aumento da expectativa de vida da população mundial e a elevada incidência dessas DCNTs na terceira idade, este estudo teve como objetivo avaliar a prevalência, os fatores de risco, a prevenção e o tratamento da hipertensão e da osteoporose, de modo a contribuir na busca por um envelhecimento saudável.

\section{Métodos}

O presente estudo trata-se de uma revisão de literatura, de caráter descritivo e de natureza qualitativa. Foi realizada uma busca na base de dados da Biblioteca Virtual em Saúde (BVS) e Periódicos da Coordenação de Aperfeiçoamento de Pessoal de Nível Superior (Capes), tendo como descritores: "doença crônica" ("chronic disease"), "idoso" ("elderly") e "hipertensão" ("hypertension") ou "osteoporose" ("osteoporosis"), com corte temporal de 10 anos, entre o período de janeiro de 2004 e dezembro de 2014. Para os critérios de inclusão desta pesquisa foram selecionados artigos originais de delineamento experimental ou observacional e artigos de revisão disponíveis on-line na íntegra e gratuitamente em português ou em inglês. Foram excluídos do trabalho artigos publicados em revistas pagas, artigos cujo o tema era diferente do proposto nesse trabalho e artigos com a data de publicação diferente da corte temporal definida no estudo. A coleta de dados foi realizada no mês de janeiro de 2015 . 
A análise dos artigos coletados realizou-se em três etapas. Na primeira etapa, foram lidos todos os títulos dos artigos encontrados nas bases de dados com os descritores referidos anteriormente. Posteriormente, à seleção dos artigos, partiu-se para a segunda etapa, que foi constituída da leitura dos resumos. $\mathrm{Na}$ terceira e última etapa de seleção, foram lidos e avaliados os textos integrais dos artigos selecionados na segunda etapa. Destaca-se que foram contabilizados apenas uma vez os artigos repetidos nas bases de dados.

\section{Resultados e discussão}

Foram encontrados 1393 artigos, 65 artigos da base de dados dos Periódicos Capes e 1328 artigos da Biblioteca Virtual em Saúde (BVS). Com base na leitura dos títulos, selecionaram-se 74 artigos, dos quais foram lidos os resumos e então selecionados os artigos para serem analisados na íntegra. Foram selecionados para o estudo 26 artigos de interesse, apresentados na tabela 1 . Além desses, foram incluídos dois manuais do Ministério da Saúde do Brasil e um boletim informativo da Organização Mundial da Saúde.

De forma a sistematizar os resultados encontrados, a discussão e a análise dos artigos foram organizadas em duas categorias temáticas: hipertensão arterial e osteoporose. 
Tabela 1 - Síntese dos artigos utilizados na revisão deste estudo.

Continua

\begin{tabular}{|c|c|c|c|c|}
\hline Autor (ano) & Objetivo & $\begin{array}{l}\text { Tipo de } \\
\text { estudo }\end{array}$ & Amostra & Principais resultados \\
\hline $\begin{array}{l}\text { Bastos-Barbosa et al. } \\
\text { (2012) }\end{array}$ & $\begin{array}{l}\text { Avaliar e comparar a taxa } \\
\text { de adesão ao tratamento } \\
\text { da hipertensão arterial } \\
\text { por diferentes métodos, } \\
\text { para estimar a taxa de } \\
\text { controle da PA, e obser- } \\
\text { var se há uma associação } \\
\text { entre controle da pressão } \\
\text { arterial e adesão. }\end{array}$ & \begin{tabular}{|l|} 
Descritivo, \\
transversal e \\
quantitativo
\end{tabular} & 60 idosos & $\begin{array}{l}\text { - Os pacientes relataram uma ingestão de } 4,5 \pm 2,3 \text { medicamen- } \\
\text { tos por dia; } \\
\text { - } 48 \% \text { informaram que frequentemente esqueciam-se de tomar } \\
\text { uma das medicações prescritas; } \\
\text { - Não houve diferenças no controle da PA entre pacientes com } \\
\text { adesão e sem adesão; }\end{array}$ \\
\hline Boeing et al. (2012) & $\begin{array}{l}\text { Avaliar as evidências } \\
\text { cientificas sobre o con- } \\
\text { sumo de legumes e } \\
\text { frutas na prevenção de } \\
\text { doenças crônicas }\end{array}$ & $\begin{array}{l}\text { Revisão da } \\
\text { literatura }\end{array}$ & 298 artigos & $\begin{array}{l}\text { - O aumento do consumo de frutas e vegetais reduzem o risco } \\
\text { de osteoporose, demência e certas doenças nos olhos; reduz } \\
\text { também o risco de hipertensão e AVC; } \\
\text { - O aumento do consumo de vegetais e frutas podem contribuir } \\
\text { para a prevenção de DPOC, asma e artrite reumatoide. }\end{array}$ \\
\hline Ebeling (2014) & $\begin{array}{l}\text { Avaliar as evidências } \\
\text { cientificas sobre os ní- } \\
\text { veis séricos efetivos de } \\
\text { vitamina } D \text {. }\end{array}$ & $\begin{array}{l}\text { Revisão } \\
\text { bibliográfica }\end{array}$ & 53 artigos & $\begin{array}{l}\text { - Não há evidências de que a vitamina } D \text { isolada tenha efeitos be- } \\
\text { néficos na densidade mineral óssea; } \\
\text { - A combinação de vitamina } D \text { e cálcio evita a perda óssea; } \\
\text { - Para manutenção a longo prazo (até } 5 \text { anos), a combinação de } \\
\text { cálcio de vitamina D é melhor que cálcio sozinho. }\end{array}$ \\
\hline $\begin{array}{l}\text { Figueiredo e Asakura } \\
(2010)\end{array}$ & $\begin{array}{l}\text { Caracterizar pacientes } \\
\text { hipertensos e conhecer } \\
\text { suas principais dificul- } \\
\text { dades para aderir ao } \\
\text { tratamento proposto } \\
\text { pela equipe de saúde }\end{array}$ & $\begin{array}{l}\text { Estudo } \\
\text { descritivo } \\
\text { de coorte } \\
\text { transversal }\end{array}$ & $\begin{array}{l}54 \text { pacientes hiper- } \\
\text { tensos }\end{array}$ & $\begin{array}{l}\text { - 66,7\% pacientes eram do sexo feminino; } \\
\text { - A pressão arterial (mmHg) sistólica e diastólica média foi } 138 \text { e } \\
\text { 83, respectivamente, mas apenas metade dos pacientes apre- } \\
\text { sentava PA controlada; } \\
\text { - A principal dificuldade relatada foi seguir dieta hipossódica; } \\
\text { - Comparando-se as orientações prescritas com aquelas que os pa- } \\
\text { cientes referiram ter recebido, houve concordância em } 37 \text { casos. }\end{array}$ \\
\hline Gilsogamo et al. (2008) & $\begin{array}{l}\text { Determinar o conjunto } \\
\text { de fatores que interfe- } \\
\text { rem na adesão ao tra- } \\
\text { tamento de pacientes } \\
\text { com hipertensão arterial } \\
\text { sistêmica atendidos em } \\
\text { um serviço especia- } \\
\text { lizado: NAHI (Núcleo } \\
\text { de Atendimento ao } \\
\text { Hipertenso) e no PSF } \\
\text { (Programa de Saúde da } \\
\text { Família) no municipio } \\
\text { de Barbacena. }\end{array}$ & $\begin{array}{l}\text { Estudo } \\
\text { de coorte } \\
\text { transversal }\end{array}$ & $\begin{array}{l}154 \text { questionários no } \\
\text { NAHI e } 246 \text { no PSF }\end{array}$ & $\begin{array}{l}\text { - } 101(5,2 \%) \text { pacientes foram incluídos na categoria não aderente } \\
\text { e } 299(74,7 \%) \text { no grupo que adere ao tratamento; } \\
\text { - Cerca de } 71 \% \text { dos pacientes afirmam ter dificuldade na aquisi- } \\
\text { ção dos medicamentos; } \\
\text { - Aproximadamente, } 50 \% \text { dos entrevistados descontinuaram o } \\
\text { tratamento no primeiro ano; } \\
\text { - } 30 \% \text { afirmam dificuldade ao acesso as consultas dizendo ser } \\
\text { muito difícil e outros } 25 \% \text {, ser dificil; } \\
\text { - Sobre a dieta, } 49,5 \% \text { dos que não aderem informam não seguir } \\
\text { as orientações, contrapondo com } 35,5 \% \text { dos pacientes incluídos } \\
\text { no grupo que adere; } \\
\text { - A adesão ao tratamento nas unidades de PSF foi maior quando } \\
\text { comparado ao centro especializado (NAHI); } \\
\text { - Quanto à assiduidade as reuniões sobre hipertensão na uni- } \\
\text { dade de tratamento, } 74,3 \% \text { dos pacientes que não seguem o } \\
\text { tratamento informam que não frequentam as palestras enquanto } \\
40,8 \% \text { dos aderentes comparecem as reuniões. }\end{array}$ \\
\hline $\begin{array}{l}\text { Kurra, Fink e Siris } \\
(2014)\end{array}$ & $\begin{array}{l}\text { Explorar a interação } \\
\text { entre diabetes e saúde } \\
\text { óssea. }\end{array}$ & $\begin{array}{l}\text { Revisão de } \\
\text { literatura }\end{array}$ & 59 artigos & $\begin{array}{l}\text { - A fratura associada à osteoporose é uma importante complica- } \\
\text { ção em pacientes com diabetes; } \\
\text { - Evidências recentes mostram que o diabetes tipo } 1 \text { e o tipo } 2 \\
\text { estão associados a um aumento do risco de fraturas. }\end{array}$ \\
\hline Landim et al. (2011) & $\begin{array}{l}\text { Verificar a adesão ao } \\
\text { tratamento farmacológi- } \\
\text { co pelos pacientes que } \\
\text { faziam uso de dois ou } \\
\text { mais anti-hipertensivos. }\end{array}$ & $\begin{array}{l}\text { Estudo } \\
\text { descritivo } \\
\text { quantitativo }\end{array}$ & $\begin{array}{l}33 \text { pacientes com hi- } \\
\text { pertensão que faziam } \\
\text { uso de dois ou mais } \\
\text { anti-hipertensivos }\end{array}$ & $\begin{array}{l}\text { - } 15 \text { pacientes tomavam três comprimidos diários, } 12 \text { pacientes to- } \\
\text { mavam o medicamento de forma diferente da prescrição médica; } \\
\text { - Quase todos os pacientes que tomavam cinco ou mais comprimi- } \\
\text { dos não faziam uso da medicação conforme prescrito; } \\
\text { - Quanto maior o número de medicamentos prescritos, menor a } \\
\text { ocorrência do seguimento correto do tratamento. }\end{array}$ \\
\hline Levis e Lagari (2012) & $\begin{array}{l}\text { Verificar os estudos epi- } \\
\text { demiológicos, observa- } \\
\text { cionais e de intervenção } \\
\text { do efeito da dieta sobre } \\
\text { a saúde óssea em seres } \\
\text { humanos. }\end{array}$ & $\begin{array}{l}\text { Revisão de } \\
\text { literatura }\end{array}$ & 48 artigos & $\begin{array}{l}\text { - O uso excessivo de álcool e uma dieta hipercalórica estão asso- } \\
\text { ciadas a menor massa óssea e maiores taxas de fratura; } \\
\text { - Os estudos confirmam o papel positivo de uma dieta saudável } \\
\text { para manter a saúde óssea; } \\
\text { - As evidências recentes sugerem que as vitaminas C e K também } \\
\text { podem ter um efeito positivo na saúde óssea. }\end{array}$ \\
\hline
\end{tabular}




\section{Continua}

\begin{tabular}{|c|c|c|c|c|}
\hline Lima et al. (2009) & $\begin{array}{l}\text { Descrever as caracte- } \\
\text { rísticas dos pacientes } \\
\text { submetidos à revascula- } \\
\text { rização do miocárdio em } \\
\text { um hospital de cardiolo- } \\
\text { gia de Fortaleza-Ceará. }\end{array}$ & \begin{tabular}{|l|} 
Estudo \\
descritivo
\end{tabular} & $\begin{array}{l}146 \text { pacientes pós ci- } \\
\text { rúrgicos de revascu- } \\
\text { larização miocárdica }\end{array}$ & $\begin{array}{l}\text { - As características sociodemográficas predominantes foram: } \\
\text { sexo masculino }(62,8 \%) \text {; idade }>60 \text { anos }(68,0 \%) \text {; baixa escola- } \\
\text { ridade }(73,1 \%) \text {; renda familiar de até um salário mínimo }(55,1 \%) \\
\text { e presença de antecedentes familiares com doenças isquêmicas } \\
\text { cardiacas }(65,4 \%) \text {. }\end{array}$ \\
\hline $\begin{array}{l}\text { Lima-Costa, Firmo e } \\
\text { Uchôa (2011) }\end{array}$ & $\begin{array}{l}\text { Investigar a incidência e } \\
\text { preditores de saúde em } \\
\text { uma população idosa } \\
\text { com baixo nivel socioe- } \\
\text { conômico. }\end{array}$ & $\begin{array}{l}\text { Estudo de } \\
\text { coorte }\end{array}$ & 1606 participantes & $\begin{array}{l}\text { - A condição de saúde mais comum foi hipertensão }(61,5 \%) \text {, se- } \\
\text { guida de sintomas crônicos do joelho ou da mão }(43,6 \%) \text {, trans- } \\
\text { tornos mentais }(38,5 \%) \text {, infecção por T. cruzi }(38,1 \%) \text { e insônia } \\
(36,7 \%) \text {. }\end{array}$ \\
\hline $\begin{array}{l}\text { Malavazi Junior, Willers } \\
\text { e Lopes (2013) }\end{array}$ & $\begin{array}{l}\text { Revisar a fisiopatologia } \\
\text { da osteoporose, bem } \\
\text { como seus fatores } \\
\text { etiológicos e incidência } \\
\text { populacional; e verifi- } \\
\text { car os benefícios que } \\
\text { o exercício físico pode } \\
\text { promover na prevenção } \\
\text { e no tratamento da oste- } \\
\text { oporose. }\end{array}$ & Bibliográfico & 35 artigos & $\begin{array}{l}\text { - O exercício físico praticado regularmente, reduz a perda de mas- } \\
\text { sa óssea, diminui a deterioração do osso, aumenta a estrutura } \\
\text { óssea e remodela; } \\
\text { - A prática do exercício melhora a qualidade de vida do idoso e é } \\
\text { utilizada como tratamento para vários tipos de doenças. }\end{array}$ \\
\hline Malta et al. (2012) & $\begin{array}{l}\text { Analisar os fatores de } \\
\text { risco e proteção para } \\
\text { Doenças Crônicas Não } \\
\text { Transmissíveis nas ca- } \\
\text { pitais do Brasil. }\end{array}$ & \begin{tabular}{|l|} 
Inquérito \\
telefônico
\end{tabular} & 54.353 entrevistas & $\begin{array}{l}\text { - O estudo mostrou diferenças na prevalência de fatores de risco e } \\
\text { proteção de DCNT entre sexos, idade e escolaridade; } \\
\text { - Os homens apresentaram maiores frequências de fatores de } \\
\text { risco como fumo, excesso de peso, consumo de refrigerantes, } \\
\text { carnes com excesso de gordura e bebidas alcoólicas; } \\
\text { - As mulheres se alimentam melhor e referem mais diagnóstico } \\
\text { médico de doenças, como hipertensão arterial, dislipidemia e } \\
\text { osteoporose; } \\
\text { - Em geral, os fatores de risco são mais frequentes na população } \\
\text { de menor escolaridade. }\end{array}$ \\
\hline Mirsaidi et al. (2014) & $\begin{array}{l}\text { Avaliar a capacidade } \\
\text { das células de estroma } \\
\text { derivadas de adipose } \\
\text { (ASC) como uma es- } \\
\text { tratégia de tratamento } \\
\text { para osteoporose rela- } \\
\text { cionada à idade }\end{array}$ & \begin{tabular}{|l|} 
Abordagens \\
in vitro e in \\
vivo
\end{tabular} & $\begin{array}{l}\text { Pacientes humanos } \\
(>65 \text { anos de idade) } \\
\text { e animais (ratos }- \\
\text { machos e fêmeas de } \\
18 \text { semanas) }\end{array}$ & $\begin{array}{l}\text { - Obteve melhora significativa no potencial osteogênico das célu- } \\
\text { las de estroma de medula óssea humana derivadas do paciente } \\
\text { osteoporóticas (BMSCs), observada quando diferenciadas em } \\
\text { meios de cultura condionados colhidos a partir de ASC humanas } \\
\text { derivadas de pacientes com osteoporose; } \\
\text { - O estudo apoia o uso de ASC como uma abordagem baseada } \\
\text { em células para tratamento da osteoporose. }\end{array}$ \\
\hline Mizokami et al. (2012) & $\begin{array}{l}\text { Analisar as doenças } \\
\text { prevalentes na popula- } \\
\text { ção idosa com relação } \\
\text { as drogas e a polifarmá- } \\
\text { cia prescrita. }\end{array}$ & \begin{tabular}{|l|} 
Estudo \\
descritivo, \\
transversal e \\
quantitativo
\end{tabular} & $\begin{array}{l}1768 \text { idosos (>65 } \\
\text { anos) hospitalizados }\end{array}$ & $\begin{array}{l}\text { - Entre os } 1768 \text { pacientes, a idade média foi de } 78 \text { anos; } \\
\text { - O número médio de doenças foi de } 7,7 \text { e o número médio de } \\
\text { fármacos foi de } 4,9 \text {; } \\
\text { - A prevalência de polifarmácia foi de hipertensão, hiperlipidemia } \\
\text { e úlcera gástrica. }\end{array}$ \\
\hline Montalcini et al. (2013) & $\begin{array}{l}\text { Explorar possiveis cau- } \\
\text { sas de perda óssea e } \\
\text { fraturas em indivíduos } \\
\text { afetados por doença } \\
\text { inflamatória cônica e } \\
\text { sugerir novos alvos } \\
\text { para intervenção. }\end{array}$ & \begin{tabular}{|l|} 
Revisão \\
bibliográfica
\end{tabular} & 90 artigos & $\begin{array}{l}\text { - Pacientes com doença inflamatória crônica, podem estar em alto } \\
\text { risco de fraturas devido a perda óssea como consequência da } \\
\text { desnutrição, causada por inflamação e mudança hormonal; } \\
\text { - Existe um novo agente, o Denosumab, que se mostrou eficaz no } \\
\text { aumento da DMO e reduzindo o risco de fraturas em pacientes } \\
\text { com osteoporose; } \\
\text { - O Denosumab é um anticorpo monoclonal, que é capaz de inibir } \\
\text { a atividade dos osteoclastos. }\end{array}$ \\
\hline Monteiro et al. (2010) & 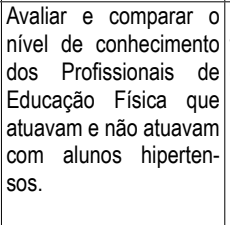 & \begin{tabular}{|l|} 
Descritivo, \\
transversal e \\
quantitativo
\end{tabular} & $\begin{array}{l}400 \text { profissionais de } \\
\text { Educação Física }\end{array}$ & $\begin{array}{l}\text { - Dos profissionais que atuavam, } 185 \text { (61,3\%) baseavam-se na } \\
\text { informação do aluno e } 117 \text { ( } 38,7 \%) \text { solicitavam a apresentação } \\
\text { de exames comprobatórios do diagnóstico; } \\
\text { - Ambos os profissionais não apresentaram um conhecimento sa- } \\
\text { tisfatório sobre os tipos de exames para o diagnóstico da hiper- } \\
\text { tensão arterial, tipos de hipertensão dos seus alunos, situações } \\
\text { que contraindicavam a atividade física e mensuração da pressão } \\
\text { arterial antes e depois da atividade física. }\end{array}$ \\
\hline
\end{tabular}


Continua

\begin{tabular}{|c|c|c|c|c|}
\hline $\begin{array}{l}\text { Nilson, Jaime e Resen- } \\
\text { de (2012) }\end{array}$ & $\begin{array}{l}\text { Apresentar a experiên- } \\
\text { cia brasileira na cons- } \\
\text { trução e implementação } \\
\text { de estratégias para a } \\
\text { redução dos limites } \\
\text { máximos de sódio nos } \\
\text { alimentos processados } \\
\text { e os atores sociais en- } \\
\text { volvidos. }\end{array}$ & \begin{tabular}{|l|} 
Relato de ex- \\
periência
\end{tabular} & $\begin{array}{l}\text { Setor público e priva- } \\
\text { do brasileiro }\end{array}$ & $\begin{array}{l}\text { - Pactuação, entre o governo e a indústria de alimentos, de metas } \\
\text { de redução dos teores máximos de sódio nos alimentos indus- } \\
\text { trializados; } \\
\text { - Realização de campanhas de educação e informação para a po- } \\
\text { pulação e elaboração das guias de redução dos teores de sódio } \\
\text { nos alimentos industrializados; } \\
\text { - Redução do consumo de sódio pela população brasileira, dos } \\
\text { atuais } 4700 \mathrm{mg} \text { para menos de } 2000 \mathrm{mg} / \text { pessoa/dia, mediante } \\
\text { diminuição do sal adicionado durante o preparo e o consumo } \\
\text { de alimentos e do sódio presente nos alimentos industrializados, } \\
\text { trabalhando eixos de comunicação, reformulação, monitoramen- } \\
\text { to e regulação; } \\
\text { - Com o fortalecimento das políticas nacionais será possivel re- } \\
\text { duzir as doenças e os óbitos associados ao consumo excessivo } \\
\text { do sódio. }\end{array}$ \\
\hline $\begin{array}{lll}\text { Oliveira } & \text { e } & \text { Moreira } \\
(2010) & & \\
\end{array}$ & $\begin{array}{l}\text { Descrever o perfil e o } \\
\text { tratamento anti-hiper- } \\
\text { tensivo não-farmaco- } \\
\text { lógico seguido por um } \\
\text { grupo de idosos porta- } \\
\text { dores de hipertensão }\end{array}$ & \begin{tabular}{|l|} 
Descritivo, \\
transversal e \\
quantitativo
\end{tabular} & 54 idosos & $\begin{array}{l}\text { - Prevalência do sexo feminino; } \\
\text { - } 66 \% \text { dos idosos apresentaram pressão arterial controlada; } \\
\text { - 74,1\% apresentaram algum grau de não-adesão à terapêutica } \\
\text { farmacológica e/ou não farmacológica; } \\
\text { - } 26 \% \text { relataram não controlar o consumo de sal na dieta diária; } \\
\text { - } 64,8 \% \text { relataram praticar atividades físicas regularmente sema- } \\
\text { nalmente. }\end{array}$ \\
\hline Oliveira et al. (2011) & $\begin{array}{l}\text { Avaliar o significado dos } \\
\text { Grupos Educativos para } \\
\text { Hipertensos na Unidade } \\
\text { Básica de Saúde de } \\
\text { São Sebastião, no mu- } \\
\text { nicípio de Juiz de Fora } \\
\text { - Minas Gerais. }\end{array}$ & \begin{tabular}{|l|}
$\begin{array}{l}\text { Estudo quali- } \\
\text { tativo }\end{array}$ \\
\end{tabular} & $\begin{array}{l}25 \text { usuários hiperten- } \\
\text { sos participantes dos } \\
\text { Grupos Educativos }\end{array}$ & $\begin{array}{l}\text { - Embora existam vulnerabilidade e deficiências nas atividades } \\
\text { preventivas, bem como seja baixa a frequência das atividades, } \\
\text { os Grupos Educativos são importantes na construção de hábitos } \\
\text { saudáveis e na adesão ao tratamento dos hipertensos na Aten- } \\
\text { ção Primária à Saúde. }\end{array}$ \\
\hline $\begin{array}{l}\text { Peterlik, Kállay e Cross } \\
\text { (2013) }\end{array}$ & $\begin{array}{l}\text { Avaliar na literatura as } \\
\text { associações existentes } \\
\text { entre o consumo inefi- } \\
\text { ciente de cálcio e o risco } \\
\text { de desenvolvimento de } \\
\text { doenças crônicas. }\end{array}$ & \begin{tabular}{|l|} 
Pesquisa sis- \\
temática (bi- \\
bliográfica)
\end{tabular} & 166 artigos & $\begin{array}{l}\text { - O receptor de detecção de cálcio (CaSR) desempenha um papel } \\
\text { importante no controle local da homeostase celular; } \\
\text { - Para um bom funcionamento dos receptores } \mathrm{Ca} 2+\text { / CaSR e vita- } \\
\text { mina } \mathrm{D} \text {, é necessário a restauração de um estado adequado de } \\
\text { cálcio e vitamina } \mathrm{D} \text {; } \\
\text { - O comprometimento do } \mathrm{Ca}^{\mathrm{A}} \text { sinalização }{ }^{2+} \text { / CaSR pode contribuir } \\
\text { para a formação inadequada do osso, hipertensão e calcificação } \\
\text { vascular. }\end{array}$ \\
\hline $\begin{array}{l}\text { Serafim, Jesus e Pierin } \\
(2010)\end{array}$ & $\begin{array}{l}\text { Caracterizar hábitos de } \\
\text { vida e conhecimentos } \\
\text { sobre eles associando } \\
\text { variáveis estudadas } \\
\text { com o controle da hi- } \\
\text { pertensão arterial em } \\
\text { pessoas atendidas em } \\
\text { ambulatório. }\end{array}$ & \begin{tabular}{|l|}
$\begin{array}{l}\text { Estudo des- } \\
\text { critivo }\end{array}$ \\
\end{tabular} & $\begin{array}{l}511 \text { indivíduos hiper- } \\
\text { tensos em controle } \\
\text { ambulatorial }\end{array}$ & $\begin{array}{l}\text { - IMC no limite superior da faixa de sobrepeso }\left(29,04 \pm 4,35 \mathrm{~kg} / \mathrm{m}^{2}\right) \text {; } \\
\text { - } 32,9 \% \text { dos homens e } 74,1 \% \text { das mulheres tinham cintura maior } \\
\text { que } 102 \mathrm{~cm} \text { e } 88 \mathrm{~cm} \text {, respectivamente; } \\
\text { - A pressão arterial era maior que o limite caracterizador da hi- } \\
\text { pertensão arterial; } \\
\text { - } 44 \% \text { referiram tabagismo ou ex-tabagismo; } \\
\text { - } 59 \% \text { não praticavam atividade física regular; } \\
\text { - } 24 \% \text { referiram ingestão de bebida alcoólica e, nestes, a quanti- } \\
\text { dade de etanol/dia foi excessiva }(71,4 \pm 83,2 \mathrm{~g}) \text {; } \\
\text { - Apenas } 22 \% \text { dos hipertensos estavam controlados e, os que } \\
\text { conheciam que o tratamento inclui a redução de peso estavam } \\
\text { mais controlados. }\end{array}$ \\
\hline $\begin{array}{l}\text { Sowińska-Przepiera } \\
\text { al. (2011) }\end{array}$ & $\begin{array}{l}\text { Avaliar as evidências na } \\
\text { literatura sobre os efei- } \\
\text { tos da deficiência de es- } \\
\text { trogênio na mineraliza- } \\
\text { ção óssea em meninas } \\
\text { durante a adolescência. }\end{array}$ & $\begin{array}{l}\text { Revisão bi- } \\
\text { bliográfica }\end{array}$ & 65 & $\begin{array}{l}\text { - A terapia hormonal deve ter um acompanhamento junto com } \\
\text { uma alimentação adequada e modificações na atividade física; } \\
\text { - A administração de oestroprogestagens no tratamento de baixa } \\
\text { DMO e distúrbios menstruais resulta em hemorragias menstruais; } \\
\text { - A puberdade é o período ideal para modificar fatores ambientais } \\
\text { associados ao ganho de massa óssea. }\end{array}$ \\
\hline Tsoi et al. (2014) & $\begin{array}{l}\text { Determinar as con- } \\
\text { dições de saúde e a } \\
\text { prescrição medicamen- } \\
\text { tosa entre os pacientes } \\
\text { com } 85 \text { anos ou mais, } \\
\text { e descrever a comple- } \\
\text { xidade clínica desses } \\
\text { pacientes. }\end{array}$ & $\begin{array}{l}\text { Revisão re- } \\
\text { trospectiva }\end{array}$ & $\begin{array}{l}564 \text { idosos com } 85 \\
\text { anos ou mais }\end{array}$ & $\begin{array}{l}\text { - A maioria dos idosos apresentava doenças cardiovasculares } \\
\text { ( } 79 \%) \text { e disfunções ósseas }(65 \%) \text {; } \\
\text { - A hipertensão (65\%) foi a condição de saúde mais prevalente } \\
\text { entre os idosos; } \\
\text { - Nas mulheres idosas, predominavam disfunções ósseas e hipo- } \\
\text { tiroidismo, enquanto a doença arterial coronariana e a diabetes } \\
\text { tipo } 2 \text { eram mais prevalentes nos homens; } \\
\text { - Os medicamentos mais prescritos entre os idoso foram atorvas- } \\
\text { tatina (33\%) e aspirina } 81 \mathrm{mg}(33 \%) \text {. }\end{array}$ \\
\hline
\end{tabular}


Conclusão

\begin{tabular}{|c|c|c|c|c|}
\hline Tu et al. (2014) & \begin{tabular}{|l} 
Examinar o resultado \\
clínico e efeito sobre \\
a fusão óssea de um \\
pós-operatório e uma \\
infusão intravenosa \\
anual de ZOL $5 \mathrm{mg}$ após \\
a cirurgia de fusão inter- \\
coronal espinhal lombar \\
em pacientes com oste- \\
oporose.
\end{tabular} & $\begin{array}{l}\text { Artigo de } \\
\text { pesquisa }\end{array}$ & $\begin{array}{l}64 \text { pacientes com es- } \\
\text { pondilolistose lombar } \\
\text { degenerativa e oste- } \\
\text { oporose submetidos } \\
\text { a LIFS }\end{array}$ & $\begin{array}{l}\text { - A incidência de fraturas de compressão vertebral e afrouxamento } \\
\text { de parafuso do pedículo foram menores no grupo ZOL do que } \\
\text { no grupo controle; } \\
\text { - Não houve diferença significativa na DMO entre os dois grupos; } \\
\text { - O tratamento com ZOL tem efeitos benéficos sobre o LIFS instru- } \\
\text { mentado tanto radiograficamente como clinicamente. }\end{array}$ \\
\hline Vilaça et al. (2011) & $\begin{array}{l}\text { Avaliar a associação } \\
\text { do estado nutricional } \\
\text { com a força muscular } \\
\text { de preensão manual e } \\
\text { a densidade mineral ós- } \\
\text { sea em idosos do sexo } \\
\text { masculino. }\end{array}$ & $\begin{array}{l}\text { Artigo de } \\
\text { pesquisa }\end{array}$ & $\begin{array}{l}41 \text { idosos do sexo } \\
\text { masculino }\end{array}$ & $\begin{array}{l}\text { - O IMC, massa magra, massa gorda e a força muscular de pre- } \\
\text { ensão manual foram menores no grupo dos idosos desnutridos; } \\
\text { - A presença de osteoporose foi maior no grupo dos idosos desnu- } \\
\text { tridos com relação aos idosos eutrófico; } \\
\text { - A cada diminuição do IMC, há diminuição também da força mus- } \\
\text { cular de preensão manual. }\end{array}$ \\
\hline
\end{tabular}

\section{Hipertensão Arterial}

\section{Prevalência}

A hipertensão arterial é uma doença multifatorial que pode acarretar graves consequências para o indivíduo, sendo considerada como a causa mais frequente de morbimortalidade nos idosos (OLIVEIRA; MOREIRA, 2010).

Tsoi et al. (2014) observou, em uma revisão de prontuários médicos realizada no Canadá com pacientes com 85 anos de idade ou mais, que a hipertensão $(65 \%)$ foi a segunda DCNT mais prevalente na população em estudo, atrás apenas das doenças cardiovasculares (79\%).

Dados apontam que $60 \%$ das pessoas entre 60 e 69 anos são acometidas por essa doença, porém em indivíduos com idade superior a 75 anos essa prevalência aumenta para 75\% (SOCIEDADE BRASILEIRA DE CARDIOLOGIA, 2010).

Malta et al. (2012), mostrou que em uma população de homens e mulheres idosas, as mulheres apresentavam maior diagnóstico médico de hipertensão, dislipidemias e osteoporose.

Além disso, observou-se que a hipertensão era mais prevalente em populações idosas de baixo nível socioeconômico (Lima-Costa, Firmo e Uchôa, 2011) e que a incidência era maior nas regiões metropolitanas em relação às regiões rurais (SOCIEDADE BRASILEIRA DE CARDIOLOGIA, 2010).

\section{Fatores de risco}

A hipertensão é herdada dos pais em 90\% dos casos, porém, há vários fatores que podem influenciar os níveis de pressão arterial, tais como: consumo de bebidas alcoólicas, fumo, grande consumo de sal, obesidade, estresse, sedentarismo e níveis elevados de colesterol. É importante que o indivíduo cuide de sua saúde durante todos os seus ciclos de vida para evitar doenças crônicas que trazem como problema secundário as doenças cardiovasculares (BRASIL, 2004).

Em estudo realizado por Malta et al. (2012), observou-se diferenças na 
prevalência de fatores de risco das DCNT entre sexos, idade e escolaridade. Os homens apresentavam maiores frequências de fatores de risco como fumo. Já as mulheres referiam menor prática de exercícios físicos.

A hipertensão arterial é considerada um dos mais importantes fatores de risco para o desenvolvimento das doenças cardiovasculares (DCV), as quais são apontadas como a quinta causa de óbito em todo o mundo (LIMA et al., 2009). No Brasil, em 2006, ocorreram 302.682 óbitos por agravos do aparelho circulatório, correspondendo a $29,4 \%$ do total de mortes (SERAFIM; JESUS; PIERIN, 2010).

\section{Prevenção}

Para o diagnóstico de hipertensão os exames mais solicitados são a mensuração da pressão arterial (PA) e teste ergométrico. Segundo as IV Diretrizes Brasileiras de Hipertensão, a medida da PA é a melhor forma para o estabelecimento do diagnóstico desta doença e a avaliação da eficácia do tratamento (SOCIEDADE BRASILEIRA DE CARDIOLOGIA, 2010). O exame de urina, creatinina, potássio sérico, colesterol total, glicemia em jejum e eletrocardiograma de repouso também são testes usados para avaliar essa doença (MONTEIRO et al., 2010).

A hipertensão é considerada uma doença silenciosa, por isso é necessário aferir a pressão arterial pelo menos uma vez ao ano, porém, se o indivíduo tem pré-disposição a ter hipertensão, é necessário seguir as recomendações médicas em relação ao número de vezes para verificar a pressão arterial durante o ano (BRASIL, 2006).

Um dos pontos importantes para prevenção da pressão arterial é ter uma dieta balanceada, saudável e pobre em sal. A recomendação da OMS quanto à ingestão diária do sal, para adultos, é no máximo $5 \mathrm{~g}$ equivalendo a $2000 \mathrm{mg}$ de sódio (ORGANIZAÇÃO MUNDIAL DA SAÚDE, 2003). Muitas evidências relacionam o consumo excessivo de sal ao desenvolvimento de doenças crônicas (NILSON; JAIME; RESENDE, 2012).

Além disso, Boeing et al. (2012) relatam a existência de evidências consistentes entre o aumento do consumo de vegetais e frutas e a redução do risco de doenças como a hipertensão arterial e doenças cardiovasculares.

\section{Tratamento}

Oliveira e Moreira (2010) descreveram o tratamento seguido por um grupo de 54 idosos com hipertensão, 74,1\% não apresentaram adesão farmacológica. Verificou que $26 \%$ não controlavam o consumo de sal e $20 \%$ também não controlavam o consumo de gordura animal ou vegetal da dieta diária, 51,9\% fizeram uso de algum tipo de preparo a base de plantas medicinais, $64,8 \%$ relatavam praticar atividade física semanalmente e $35 \%$ não fazem nenhum tipo de exercício físico.

De acordo com Bastos-Barbosa et al. (2012), a não adesão ao tratamento é identificada como a causa principal da pressão arterial sistólica não controlada, representando assim um risco significativo de eventos cardiovasculares. 
A OMS estimou que $50 \%$ a $70 \%$ dos pacientes não aderem ao tratamento prescrito (ORGANIZAÇÃO MUNDIAL DA SAÚDE, 2003). É imprescindível identificar as dificuldades com vistas a uma melhor adesão ao tratamento da hipertensão, bem como das doenças crônicas associadas, reforçando a necessidade de se informar e discutir as condições observadas pelos profissionais da saúde (FIGUEIREDO; ASAKURA, 2010).

Segundo Landim et al. (2011), os fatores que colaboram para não adesão ao tratamento, estão relacionados com o número de fármacos a serem tomados, causando confusão e esquecimento do medicamento, sendo mais difícil o regime terapêutico para os idosos, pois utilizam mais medicamentos que qualquer outro grupo etário e possuem menor habilidade para apreender e adquirir informações. De acordo com Mizokami et al. (2012), as medicações anti-hipertensivas estão entre as mais administradas entre os idosos.

Uma das maneiras mais eficientes para estimular a adesão ao tratamento da hipertensão arterial é a educação em saúde. Nesse sentido, a abordagem multiprofissional, por meio dos diversos saberes dos profissionais envolvidos e a realização de grupos educativos têm se mostrado instrumentos de grande valor no controle da doença hipertensiva, por se tratar de uma forma de interação entre profissionais e usuários, fazendo com que estes possam refletir e expor a sua realidade, observar os problemas mais comuns entre eles, trocar experiências e propor mudanças de hábitos (OLIVEIRA et al., 2011).

De acordo com as VI Diretrizes Brasileiras de Hipertensão, a hipertensão é considerada um dos principais fatores de risco modificáveis e um dos mais importantes problemas de saúde pública. A não adesão ao tratamento pode trazer sérias consequências ao paciente. Dessa forma, destaca-se a importância da equipe multidisciplinar no acompanhamento do tratamento da hipertensão no idoso, o que contribui para a melhor qualidade de vida do paciente e principalmente para o aumento da adesão ao tratamento anti-hipertensivo (SOCIEDADE BRASILEIRA DE CARDIOLOGIA, 2010).

O tratamento para portadores de hipertensão é de suma importância para a prevenção de complicações. Fundamenta-se no uso de medicamentos anti-hipertensivos e em medidas não farmacológicas, o primeiro tem por base o uso de fármacos prescritos pelo médico, conforme a gravidade do quadro. Já o segundo é feito por meio do controle do peso, da melhora do padrão alimentar, da redução do consumo do sal, da moderação no consumo de bebidas alcoólicas, da prática regular de exercício físico, da abstenção do tabagismo e do controle do estresse psicoemocional, sendo indicado para todos os hipertensos e normotensos (GILSOGAMO et al., 2008; SOCIEDADE BRASILEIRA DE CARDIOLOGIA, 2010).

A hipertensão arterial, considerada a causa mais frequente de morbimortalidade nos idosos, é uma doença mais pre- 
valente entre mulheres, as quais referem menor prática de exercício físico e entre indivíduos de menor escolaridade, sendo mais prevalente nas regiões metropolitanas em relação as regiões rurais. Entre os fatores de risco da hipertensão tem-se a idade, o fator hereditário e o estilo de vida, estando envolvido o consumo de bebidas alcoólicas, o tabagismo, o consumo excessivo de sal, o excesso de peso, o estresse, a falta de exercício físico e os níveis elevados de colesterol. A prevenção dessa doença consiste em uma dieta balanceada rica em frutas e verduras e com valores diários inferiores a $5 \mathrm{~g}$ de sal. Além disso, outra medida preventiva é a realização da aferição da pressão arterial periodicamente. $\mathrm{O}$ tratamento da hipertensão consiste na administração de medicamentos anti-hipertensivos, no controle da alimentação e na prática de exercícios físicos regulares.

\section{Osteoporose}

\section{Prevalência}

Segundo Malavazi Junior, Willers e Lopes (2013), a osteoporose é uma doença inicialmente sem sintomas e de caráter sistêmico que pode alterar gravemente a estrutura óssea. Essa doença tem aumentado de forma significativa nos últimos anos em consequência do aumento da população idosa mundial e do estilo de vida sedentário proporcionado pelo mundo atual.

Malta et al. (2012), mostrou que em uma população de homens e mulheres idosas, as mulheres apresentavam maior diagnóstico médico de osteoporose, estando a maior ocorrência de osteoporose nessa população relacionada principalmente as mudanças hormonais ocorridas no período da menopausa.

\section{Fatores de risco}

De acordo com Kurra, Fink e Siris (2014), a idade, o sexo, o índice de massa corporal (IMC), o uso prolongado de glicocorticoides, o tabagismo, o consumo excessivo de álcool, o histórico familiar de fraturas e a baixa densidade mineral óssea (DMO) podem causar ou contribuir para a ocorrência de fraturas e osteoporose.

Em estudo realizado por Vilaça et al. (2011) com 41 idosos do sexo masculino, sendo 20 eutróficos e 21 desnutridos, avaliou-se a composição corporal, a força muscular e a ocorrência de osteoporose. Os resultados demostraram que o grupo desnutrido apresentou valores menores dos parâmetros antropométricos, de composição corporal e força muscular, e a ocorrência de osteoporose foi significativamente maior com relação ao grupo eutrófico. Desta forma, a desnutrição está associada à diminuição da DMO e da força muscular em idosos do sexo masculino.

Alguns fatores nutricionais como a deficiência de vitamina $\mathrm{D}$ e de cálcio também são considerados fatores de risco para o desenvolvimento da osteoporose (PETERLIK, KÁLLAY, CROSS, 2013).

Em estudo realizado por Montalcini et al. (2013), evidenciou-se que pacientes com doenças crônicas inflamatórias 
poderiam apresentar elevado risco para o desenvolvimento de fraturas em decorrência da má alimentação, do processo inflamatório e das mudanças hormonais.

Segundo Sowi $\square$ ska-Przepiera et al. (2011) os fatores hormonais possuem um papel importante no acúmulo de massa óssea, principalmente no período da puberdade sendo o oposto durante o climatério. Hormônios, como o estrogênio, exercem relevante função no processo da osteogênese, sendo que sua falta pode causar sérias consequências para a mineralização óssea.

\section{Prevenção}

Levis e Lagari (2012) indicam que um estado nutricional eutrófico associado ao consumo adequado de proteína e de cálcio, vitamina $\mathrm{D}$, frutas e vegetais exercem uma influência positiva sobre a saúde óssea. Já o consumo excessivo de calorias e álcool está associado à diminuição da DMO e ao aumento do risco de fraturas.

Boeing et al. (2012) também referem evidências entre o aumento no consumo de vegetais e frutas e a redução do risco do desenvolvimento de doenças visuais, demência e risco de osteoporose.

Dados atuais de ensaios bioquímicos controlados, observacionais e randomizados, indicam níveis séricos de $50 \mathrm{nmol}$. $\mathrm{l}^{\square 1}$ do receptor da vitamina D (25-hidroxivitamina D) para normalização do hormônio paratireoide (PTH) e função ideal da célula óssea. A insuficiência de 25-hidroxivitamina $\mathrm{D}$ pode trazer consequências como: hiperparatireoidismo secundário, perda de massa óssea e aumento do risco de fraturas. A ingestão de cálcio também é fundamental na melhoria do estado de vitamina $\mathrm{D}$ em pacientes com insuficiência de níveis de 25-hidroxivitamina D (EBELING, 2014).

\section{Tratamento}

O tratamento da osteoporose é muito discutido, em estudo realizado com 64 pacientes que apresentavam osteoporose submetida à Lifs (cirurgia de artrodese lombar) e espondilolistese degenerativa, dividiu-se em dois grupos, de modo que um recebeu tratamentos com infusão intravenosa de ácido zoledrônico (ZOL) e o outro não (grupo controle). Assim, pode observar que a incidência de fraturas de compressão vertebral foi menor no grupo submetido à infusão com ZOL (19\%) e maior no grupo controle (51\%). Dessa forma, o tratamento com ZOL pode ser recomendado para pacientes com osteoporose submetidos à LIFS (TU et al., 2014).

Outra estratégia para o tratamento é o uso de células estromais derivadas de tecido adiposo (ASC), que possuem capacidade rápida de sofrer diferenciação osteogênica. Foram realizadas abordagens in vitro e in vivo em ratos a base de ASC isoladas de 18 semanas de idade. Uma única injeção de intratibial ASC isogênicas melhorou significativamente a qualidade do osso trabecular, depois de 6 semanas, em comparação com os ossos contralaterais não tratados. Também induziu um aumento em vários 
marcadores moleculares de remodelação óssea (MIRSAIDI et al., 2014).

Ainda, de acordo com Montalcini et al. (2013), algumas alternativas para o tratamento da osteoporose seriam o desenvolvimento de fármacos que atuem na modulação da citocina inflamatória interleucina 6 (IL-6), uma vez que essa aumenta a atividade dos osteoclastos.

A osteoporose acomete a população idosa, principalmente as mulheres idosas. Alguns dos fatores de risco para o desenvolvimento dessa doença são a idade, o sexo, o índice de massa corporal, o uso prolongado de glicocorticoides, os níveis séricos de vitamina $\mathrm{D}$ e cálcio, o histórico familiar e a baixa densidade mineral óssea. A prevenção da osteoporose consiste na manutenção do peso e em uma dieta balanceada, principalmente de proteína, cálcio e vitamina D. O tratamento da osteoporose ainda é muito discutido, sendo que, atualmente, as técnicas consistem na aplicação de ácido zoledrônico e no desenvolvimento de fármacos que atuem na modulação de citocinas inflamatórias.

\section{Considerações finais}

A hipertensão destaca-se como a doença crônica não transmissível mais prevalente entre a população idosa. Entre os fatores de risco para o desenvolvimento da hipertensão tem-se o fator genético, o etilismo, o tabagismo, o consumo excessivo de sódio, o excesso de peso, o estresse e os níveis elevados de colesterol. A prevenção desta doença consiste na realização da aferição da pressão arterial e em uma dieta balan- ceada pobre em sal. O tratamento da hipertensão consiste na administração de medicamentos anti-hipertensivos, no controle da dieta e na prática de exercícios físicos regulares, sendo que o tratamento farmacológico, usualmente indicado para os pacientes hipertensos, apresenta baixa adesão entre os mesmos.

Já a osteoporose, doença assintomática que altera a morfologia óssea, acomete principalmente a população idosa e em especial as mulheres, devido às alterações hormonais ocorridas nesse período. Os fatores de risco para o desenvolvimento desta doença são a idade, o sexo, o índice de massa corporal, o uso prolongado de glicocorticoides, o tabagismo, o etilismo, o histórico familiar e a baixa densidade mineral óssea. Além disso, a deficiência de vitamina $\mathrm{D}$ e de cálcio também são considerados fatores de risco para o desenvolvimento da osteoporose. A prevenção desta doença consiste na manutenção do peso e em uma dieta balanceada, principalmente de proteína, cálcio e vitamina $\mathrm{D}$. O tratamento da osteoporose ainda é muito discutido, sendo que atualmente as técnicas consistem na aplicação de ácido zoledrônico e no desenvolvimento de fármacos que atuem na modulação de citocinas inflamatórias.

Uma das limitações deste estudo foi o fato de terem sido utilizados apenas artigos disponíveis online na íntegra e gratuitos, não apresentando assim dados publicados em revistas pagas. Porém, tendo em vista a coorte temporal utilizada nesta revisão, acredita-se que os artigos selecionados foram sufi- 
cientemente capazes de responder aos objetivos do estudo sobre a hipertensão e a osteoporose.

Em suma, tendo em vista o envelhecimento da população brasileira e do aumento da expectativa de vida desses idosos, tem-se o aumento na demanda de serviços de saúde especializados a essa população. A revisão de literatura realizada neste estudo auxilia no esclarecimento dos profissionais de saúde e da população sobre a prevalência, os fatores de risco, a prevenção e o tratamento da hipertensão e da osteoporose, contribuindo na busca por um envelhecimento saudável.

\section{Arterial hypertension and osteoporosis in the aging process: a literature review}

\section{Abstract}

Non-communicable chronic diseases are one of the main causes of death in Brazil. The aim of this study was to perform a literature review evaluating the prevalence, risk factors, prevention and treatment of two diseases involved in the aging process, hypertension and osteoporosis. The research was conducted in the databases BIREME and Periodicals of the Coordination for the Improvement of Higher Education Personnel (CAPES), whose search terms were "chronic disease", "elderly", "hypertension" or "osteoporosis". Were including original and reviews articles in portuguese and english, and excluding articles that were not available online for free in full. Were found 1393 articles, being selected for study 26 national and international studies published between 2004 and 2014. Results showed that hypertension and oste- oporosis consist in diseases with high prevalence among aging people, especially among women. Genetic and environmental factors, such as smoking, alcoholism, sedentary lifestyle and diet are the main risk factors for these diseases. The prevention of these diseases is based on care with risk factors such as physical exercise and a diet rich in fruits and vegetables, and low in salt, in the case of hypertension. The treatment of osteoporosis and hypertension usually occurs by surgical and pharmacological intervention, respectively. The results of this study contribute to the better understanding of these diseases, assisting in the search for healthy aging.

Keywords: Aging. Hypertension. Osteoporosis.

\section{Referências}

ANDRADE, S. A. F. Osteoporose: um problema de saúde pública. Revista UNILUS Ensino e Pesquisa, Santos, v. 12, n. 28, jul./ set. 2015.

BASTOS-BARBOSA, R. G.; FERRIOLLI, E.; MORIGUTI, J. C.; NOGUEIRA, C. B.; NOBRE, F.; UETA, J. et al. Adesão ao Tratamento e Controle da Pressão Arterial em Idosos com Hipertensão. Arquivo Brasileiro de Cardiologia, Rio de Janeiro, v. 99, n.1, p. 636-641, jul. 2012.

BOEING, H.; BECHTHOLD, A.; BUD, A.; ELLINGER, S.; HALLER, D.; KROKE, A. et al. Critical review: vegetables and fruit in the prevention of chronic diseases. European Journal of Nutrition, v. 51, n. 6, p. 637-663, jun. 2012.

BRASIL. Ministério da Saúde. Vigilância alimentar e Nutricional - SISVAN. Protocolo do SISVAN, Norma Técnica da Vigilância Alimentar e Nutricional. Brasília: Ministério da Saúde, 2004. Disponível em: <http://www. saude.gov.br/bvs>. Acesso em: jul. 2016. 
BRASIL. Ministério da Saúde. Secretaria de Atenção à Saúde. Departamento de Atenção Básica. Hipertensão arterial sistêmica. Cadernos de Atenção Básica 15. Série A. Normas e Manuais Técnicos. Brasília: Ministério da Saúde, 2006. Disponível em: <http://www. saude.gov.br/bvs>. Acesso em: 25 jul. 2016.

CAMARANO, A. A. Tratado de Geriatria e Gerontologia. Rio de Janeiro: Guanabara Koogan, 2006.

EBELING, P. R. Vitamin D and bone health: Epidemiologic studies. Bonekey Reports, Chicago, v. 5, n. 3, p. 1-5, mar. 2014.

FIGUEIREDO, N. N.; ASAKURA, L. Adesão ao tratamento anti-hipertensivo: dificuldades relatadas por indivíduos hipertensos. Acta Paulista Enfermagem, São Paulo, v. 23, n. 6, p. 782-787, 2010.

GILSOGAMO, C. A.; OLIVEIRA, J. C.; TEIXEIRA, J. C. A.; GROSSI, L. C. N.; MOREIRA, M. M. P.; DINIZ, L. O. Fatores que interferem na adesão ao tratamento da hipertensão arterial sistêmica em pacientes atendidos no Núcleo de Atendimento ao Hipertenso (NAHI) em Programa Saúde da Família (PSF), no município de Barbacena. Revista Brasileira de Medicina de Família e Comunidade, Rio de Janeiro, v. 4, n. 15, p. 179-188, out./dez. 2008.

IBGE. INSTITUTO BRASILEIRO DE GEOGRFIA E ESTATÍSTICA. Síntese de Indicadores Sociais: uma análise das condições de vida da população brasileira. Rio de Janeiro: IBGE, 2010. Disponível em: <http://www. ibge.gov.br>. Acesso em: 22 ago. 2016.

KURRA, S.; FINK, D. A.; SIRIS, E. S. Osteoporosis-associated Fracture and Diabetes. Endocrinology Metabolism Clinics of North America, v. 43, n. 1, p. 233-243, mar. 2014.

LANDIM, M. P.; OLIVEIRA, C. J.; ABREU, R. N. D. C.; MOREIRA, T. M. M.; VASCONCELOS, S. M. M. Adesão ao tratamento farmacológico anti-hipertensivo por pacientes de unidade da estratégia da família. Revista
APS, Ceará, v. 14, n. 2, p. 132-138, abr/jun. 2011.

LEVIS, S.; LAGARI, V. S. The Role of Diet in Osteoporosis Prevention and Management. Current Osteoporosis Reports, v. 10, n. 1, p. 296-302, set. 2012.

LIMA, F. E. T.; ARAÚJO, T. L.; MOREIRA, T. M. M.; LOPES, M. V. O.; MEDEIROS, A. M. Características sociodemográficas de pacientes submetidos à revascularização miocárdica em um hospital de Fortaleza-CE. Revista Rene, Fortaleza, v. 10, n. 3, p. 37-43, jul/set. 2009.

LIMA-COSTA, M. F.; FIRMO, J. O. A.; UCHÔA, E. The Bambuí Cohort Study of Aging: methodology and health profile of participants at baseline. Cadernos de Saúde Pública, Rio de Janeiro, v. 27, n. 3, p. 53275335, 2011.

MACHADO, W. D.; GOMES, D. F.; FREITAS, C. A. S. L.; BRITO, M. C. C.; MOREIRA, A. C. A. Idosos com doenças crônicas não transmissíveis: um estudo em grupos de convivência. ReonFacema, v. 3, n. 2, p. 444451, abr/jun. 2017.

MALAVAZI JUNIOR, I.; WILLERS, C. D. A.; LOPES, C. P. B. A influência do exercício físico na prevenção e tratamento da osteoporose em idosos. Revista Saúde e Pesquisa, Maringá, v. 6, n. 3, p. 487-494, set/dez. 2013.

MALTA, D. C.; SILVA, S. A.; OLIVEIRA, P. P. V.; ISER, B. P. M.; BERNAL, R. T. I.; SARDINHA, L. M. V. et al. Resultado do monitoramento dos fatores de risco e proteção para doenças crônicas não transmissíveis nas capitais brasileiras por inquérito telefônico. Revista Brasileira de Epidemiologia, v. 15, n. 3, p. 639-50, 2012.

MIRSAIDI, A.; GENELIN, K.; VETSCH, J. R.; STANGER, S.; THEISS, F.; LINDTNER, R. A. et al. Therapeutic potential of adipose-derived stromal cells in age-related osteoporosis. Biomaterials, v. 35, n. 26, p. 7326-7335, ago. 2014. 
MIZOKAMI, F.; KOIDE, Y.; NORO, T.; FURUTA, K. Polypharmacy with common diseases in hospitalized elderly patients. The American Journal of Geriatric Pharmacotherapy, v. 10, n. 2, p. 123-128, abr. 2012.

MONTALCINI, T.; ROMEO, S.; FERRO, Y.; MIGLIACCIO, V.; GAZZARUSO, C.; PUJIA, A. Osteoporosis in chronic inflammatory disease: the role of malnutrition. Endocrine, v. 43 , n. 1, p. 59-64, fev. 2013.

MONTEIRO, L. Z.; SPINATO, I. L.; SILVA, C. A. B.; SANTOS, Z. M. S. A.; MONTENEGRO JUNIOR, R. M. Nível de conhecimento do Profissional de Educação Física frente a alunos com hipertensão arterial nas academias de ginástica. Revista Brasileira de Cineantropometria \& Desempenho Humano, São Paulo, v. 12, n. 4, p. 262-268, 2010.

NILSON, E. A. F.; JAIME, P. C.; RESENDE, D. O. Iniciativas desenvolvidas no Brasil para a redução do teor de sódio em alimentos processados. Revista Panamericana de Salud Pública, Brasília, v. 34, n. 4, p. 287-292, 2012.

OLIVEIRA, E. A. F.; ALMEIDA, A. B.; SOUZA, E. E. C. M.; PAULA, N. C. S.; PEREIRA, E. R.; MOREIRA, R. O. et al. Significado dos grupos educativos de hipertensão arterial na perspectiva do usuário de uma unidade de atenção primária à saúde. Revista APS, v. 14, n. 3, p. 319-326, jul./set. 2011.

OLIVEIRA, C. J.; MOREIRA, T. M. M. Caracterização do tratamento não farmacológico de idosos portadores de hipertensão arterial. Revista Rene, Fortaleza, v. 11, n. 1, p. 76-85, jan./mar. 2010.

ORGANIZAÇÃO MUNDIAL DA SAÚDE. Dieta, nutrição e prevenção de doenças crônicas. Genebra, 2003. Disponível em: <http:// who.int>. Acesso em: 07 jul. 2016.

ORGANIZAÇÃO MUNDIAL DA SAÚDE. Global status report on noncommunicable diseases 2014. Genebra, 2014. Disponível em: <http://www.who.int/nmh/publications/ ncd-status-report-2014/en/>. Acesso em: 03 mar. 2018.
PETERLIK, M.; KÁLLAY, E.; CROSS, H. S. Calcium Nutrition and Extracellular Calcium Sensing: Relevance for the Pathogenesis of Osteoporosis, Cancer and Cardiovascular Diseases. Nutrients, v. 5, n. 1, p. 302-327, jan. 2013.

REBELO, A. I. M. F. T. A osteoporose no envelhecimento. 2016. Dissertação (Mestrado Integrado em Medicina) - Faculdade de Medicina da Universidade de Coimbra, Coimbra, 2016.

SERAFIM, T. S.; JESUS, E. S.; PIERIN, A. M. G. Influência do conhecimento sobre o estilo de vida saudável no controle de pessoas hipertensas. Acta Paulista Enfermagem, São Paulo, v. 23, n. 5, p. 658-664, set./out. 2010.

SOCIEDADE BRASILEIRA DE CARDIOLOGIA. VI Diretrizes Brasileiras de Hipertensão. Arquivo Brasileiro de Cardiologia, Rio de Janeiro, v. 95, n. 1, p. 1-51, 2010.

SOWIŃSKA-PRZEPIERA, E.; ANDRYSIAKMAMOS, E.; JARZABEK-BIELECKA, G.; FRIEBE, Z.; SYRENICZ, A. Effects of oestrogen deficiency on bone mineralisation in girls during "adolescent crisis". Endokrynologia Polska, Szczecin, v. 62, n. 6, p. 538-546, 2011.

TSOI, C. S.; CHOW, J. Y.; CHOI, K. S.; LI, H. W.; NIE, J. X.; TRACY, C. S. et al. Medical characteristics of the oldest old: retrospective chart review of patients aged $85+$ in an academic primary care centre. BMC Research Notes, v. 7, n. 1, p. 340-352, jun. 2014.

TU, C. W.; HUANG, K. F.; HSU, H. T.; LI, H. Y.; YANG, S. S.; CHEN, Y. C. Zoledronic acid infusion for lumbar interbody fusion in osteoporosis. Journal of surgical research, Philadelphia, v. 192, n. 1, p. 112-116, nov. 2014.

VIEIRA, C. P. B. et al.; NASCIMENTO, J. J.; BARROS, S. S.; LUZ, M. H. B. A.; VALLE, A. R. M. C. Prevalência referida, fatores de risco e controle da hipertensão arterial em idosos. Cienc Cuid Saude, v. 15, n. 3, p. 413-420, jul./set. 2016. 
VILAÇA, K. H. C.; FERRIOLLI, E.; LIMA, N. K. C.; PAULA, F. J. A.; MARCHINI, J. S.; MORIGUTI, J. C. Força muscular e densidade mineral óssea em idosos eutróficos e desnutridos. Revista de Nutrição, Campinas, v. 24 , n. 6 , p. $845-852$, jan. 2011. 